\title{
POLYMORPHISM UNDER APOSTATIC AND APOSEMATIC SELECTION
}

\author{
VINTON THOMPSON \\ Department of Biology, Roosevelt University, 430 South Michigan Avenue, Chicago, Illinois \\ 60605 USA
}

Received $31 . \mathrm{i} .84$

\begin{abstract}
SUMMARY
Selection for warning colouration in well-defended species should lead to a single colour form in each local population, but some species are locally polymorphic for aposematic colour forms. Single-locus two-allele models of frequencydependent selection indicate that combined apostatic and aposematic selection may maintain stable polymorphism for one, two or three aposematic forms, provided that at least one form is subject to net apostatic selection. Frequencyindependent selective differences between colour forms broaden the possibilities for aposematic polymorphism but lead to monomorphism if too large. Concurrent apostatic and aposematic selection may explain polymorphism for warning colouration in a number of jumping or moderately unpalatable insects.
\end{abstract}

\section{INTRODUCTION}

Polymorphism for warning colouration poses a paradox for evolutionists because, as Fisher (1958) seems to have been the first to note, selection for warning colouration (aposematic selection) should lead to monomorphism. Under aposematic selection predators tend to avoid previously encountered phenotypes of undesirable prey, so that the fitness of each phenotype increases as it becomes more common. Given the presence of two forms, each will suffer at the hands of predators conditioned to avoid the other and one will eventually prevail because as soon as it gets the upper hand numerically it will drive the other to extinction. Nonetheless, many species are polymorphic for colour forms that appear to be aposematic. The ladybird beetles (Coccinellidae) furnish a number of particularly striking examples (Hodek, 1973; and see illustration in Ayala, 1978).

Edmunds (1969), Thompson (1973, 1974) and Greenwood et al., (1981) have suggested that some polymorphisms for warning colouration reflect a balance between aposematic and apostatic selection. Under apostatic selection predators tend to overlook rarer phenotypes of desirable prey, so that the fitness of each phenotype decreases as it becomes more common. Apostatic selection therefore promotes colour polymorphism (Clarke, 1979), suggesting that suitably strong apostatic selection might overcome the homogenising force of aposematic selection. Because both apostatic and aposematic selection lead to changes in phenotype fitness with phenotype frequency, they represent forms of frequency-dependent selection (Ayala and Campbell, 1974; Clarke, 1979).

Clarke and O'Donald (1964) and Turner $(1978,1980)$ have applied simple algebraic models of frequency-dependent selection to the analysis of apostatic and mimetic polymorphisms. Here I extend their approach to the analysis of conditions for polymorphism under combined apostatic and 
aposematic selection. The models employed below are general and may be applicable to other instances of frequency-dependent selection.

\section{General fREQUENCY-DEPENDENT SELECTION WITH DOMINANCE}

Consider a colour polymorphism with two phenotypes controlled by two alleles at one locus in an organism with discrete generations and infinite population size. Let the dominant allele $A_{1}$ occurring at frequency $p$ determine colour form 1 and let the recessive allele $A_{2}$ occurring at frequency $q$ determine colour form 2. Assume that mating is random and let the fitnesses of the genotypes take the form:

$$
\begin{array}{ccc}
\text { Genotype } & \text { Frequency } & \text { Fitness } \\
A_{1}- & 1-q^{2} & C_{1}-s_{1}\left(1-q^{2}\right) \\
A_{2} A_{2} & q^{2} & C_{2}-s_{2} q^{2}
\end{array}
$$

where $s_{1}$ and $s_{2}$ represent coefficients of frequency-dependent selection on the respective phenotypes and $C_{1}$ and $C_{2}$ represent frequency-independent selection components (i.e., the fitnesses of the respective phenotypes at the limiting frequency zero). Positive $s$ values correspond to apostatic selection, negative $s$ values to aposematic selection, and the fitnesses are linear functions of phenotype frequency (Fig. 1). When $C_{1}=C_{2}=1$ this model reduces to a model first considered by Wright (1955) and more recently by O'Donald (1980). When $C_{1}=C_{2}=1$ and $s_{1}=s_{2}$ it reduces to a model considered by Clarke and O'Donald (1964).

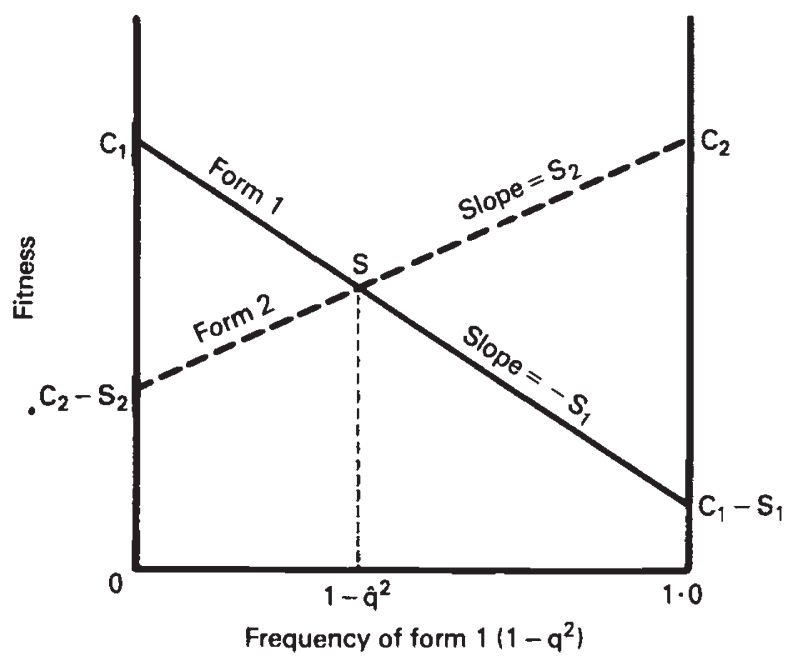

FIG. 1. Graphical representation of frequency-dependent selection on two colour forms. Solid line represents the fitness-frequency function of form 1 with fitness equal to $C_{1}-s_{1}\left(1-q^{2}\right)$. Broken line represents the fitness-frequency function of form 2 with fitness equal to $C_{2}-s_{2}+s_{2}\left(1-q^{2}\right)$ or, in simpler form, $C_{2}-s_{2} q^{2}$. Equilibrium phenotype frequency occurs where the functions intersect. In this case both forms are subject to apostatic selection, $C_{1}=C_{2}$, and there is a stable $(S)$ polymorphic equilibrium $\hat{q}$ with form 1 at frequency $1-\hat{q}^{2}$. For explanation of other symbols, see text. 
Following Lewontin (1958) and Clarke and O'Donald (1964), Fisher's (1958) general formulae can be applied to show that:

$$
\hat{q}=\left[\left(C_{2}-C_{1}+s_{1}\right) /\left(s_{1}+s_{2}\right)\right]^{1 / 2}
$$

and

$$
\Delta q=\frac{p q^{2}\left\{\left[C_{2}-s_{2} q^{2}\right]-\left[C_{1}-s_{1}\left(1-q^{2}\right)\right]\right\}}{q^{2}\left[C_{2}-s_{2} q^{2}\right]+\left(1-q^{2}\right)\left[C_{1}-s_{1}\left(1-q^{2}\right)\right]}
$$

where $\hat{q}$ is the equilibrium frequency of $A_{2}$ and $\Delta q$ is the single generation change in the frequency of $A_{2}$ given initial frequency $q$. The denominator of (3) is $\bar{W}$, the mean fitness of a population with $A_{2}$ at frequency $q$. At equilibrium the phenotypes have equal fitness.

A polymorphic equilibrium will occur provided that $\hat{q}$ lies in the range $0<\hat{q}<1$ and the fitness of the phenotypes at equilibrium is positive. Substitution of (2) for $\hat{q}$ in the inequalities $0<\hat{q}$ and $\hat{q}<1$ shows $\hat{q}$ to lie in the appropriate range under the following conditions:

$$
\begin{gathered}
s_{1}+s_{2}>0 \\
C_{1}>C_{2}-s_{2} \\
C_{2}>C_{1}-s_{1}
\end{gathered}
$$

or

$$
\begin{gathered}
s_{1}+s_{2}<0 \\
C_{1}<C_{2}-s_{2} \\
C_{2}<C_{1}-s_{1}
\end{gathered}
$$

When the sum $s_{1}+s_{2}$ is positive and less than $\hat{\hat{W}} /\left[\hat{p} \hat{q}^{3}\right]$, the equilibrium is stable (Appendix I). When the sum is negative, the equilibrium is unstable, so that the conditions defined in (4) are necessary and sufficient for stable polymorphism, provided that the sum $s_{1}+s_{2}$ is not too large.

When there are no frequency-independent selective differences $\left(C_{1}=\right.$ $C_{2}$ ), stable polymorphism can occur only if both forms are subject to apostatic selection $\left(s_{1}\right.$ and $s_{2}$ positive, fig. 1). When both forms are subject to aposematic selection ( $s_{1}$ and $s_{2}$ negative), the equilibrium is unstable (fig. 2a). When one form is subject to apostatic selection and the other to aposematic selection (one $s$ positive and one negative), or when one is subject to frequency-dependent selection and the other is not (one $s$ equal to zero), a polymorphic equilibrium cannot occur (figs. $2 b, c, d$ ).

However, when the frequency-independent fitness components of the colour forms differ $\left(C_{1} \neq C_{2}\right)$ there are broader possibilities for polymorphism. First, stable polymorphism for aposematically and apostatically selected colour forms may occur, provided that:

(a) the intensity of apostatic selection (the absolute value of $s$ ) is greater than the intensity of aposematic selection and

(b) the apostatic form has a sufficient frequency-independent advantage (fig. 2e). Second, stable polymorphism is possible for an apostatically selected form and a form that is not subject to frequency-dependent selection, provided that the apostatic form has a frequency-independent advantage (fig. 2g). Turner (1978) reaches an analogous conclusion for polymorphic Batesian mimetic forms and points out that an apparently contradictory 

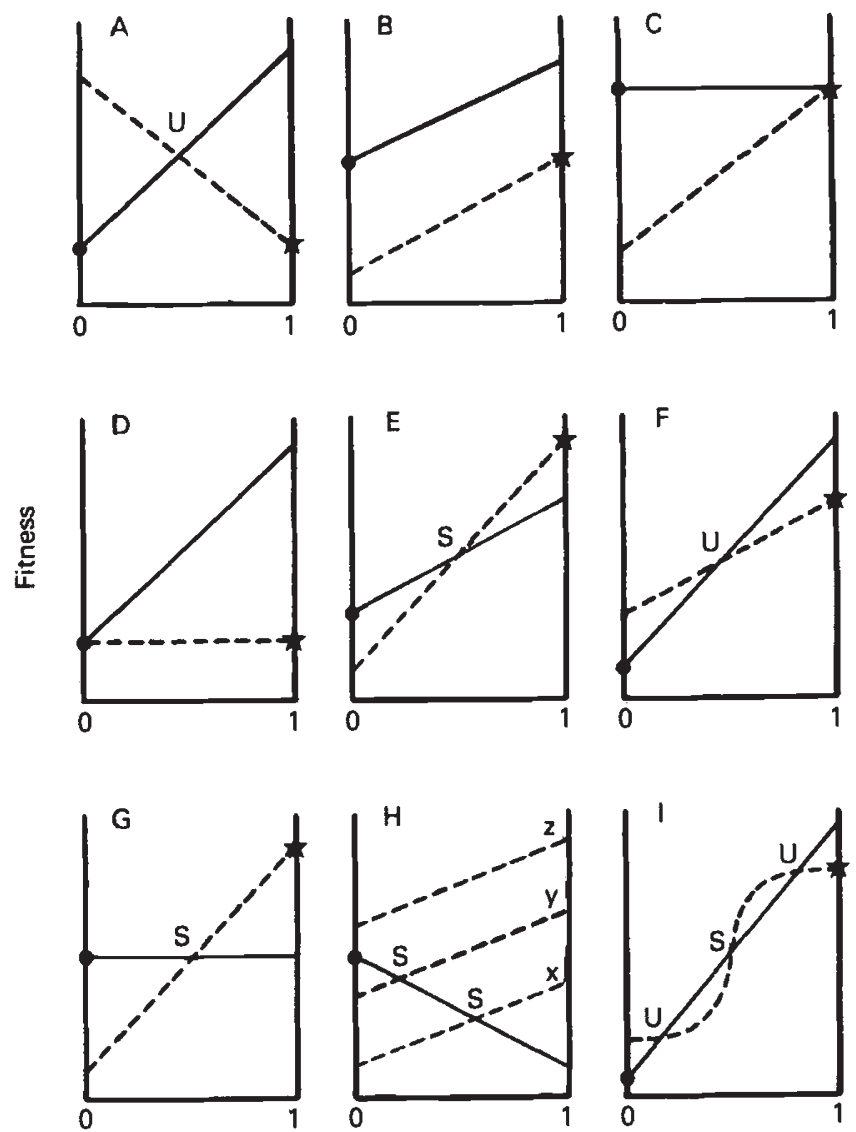

Frequency of form 1

FIG. 2. Consequences of aposematic and apostatic selection on two colour forms. As in fig. 1, a solid line represents form 1 and a broken line form 2. A dot represents $C_{1}$ while a star represents $C_{2}$. Stable polymorphic equilibrium is signified by $S$, unstable equilibrium by $U$. A. Both forms subject to aposematic selection, $C_{1}=C_{2}$. B Form 1 aposematic, form 2 apostatic, $C_{1}=C_{2}$. C. Form 1 frequency-neutral, form 2 apostatic, $C_{1}=C_{2}$. D. Form 1 aposematic, form 2 frequency-neutral, $C_{1}=C_{2}$. E. Form 1 aposematic, form 2 apostatic, $C_{1}>C_{2}-s_{2}, C_{2}>C_{1}-s_{1}$. F. Form 1 aposematic, form 2 apostatic, $C_{1}<C_{2}-s_{2}, C_{2}<$ $C_{1}-s_{1}$. G. Form 1 frequency-neutral, form 2 apostatic, $C_{1}>C_{2}-s_{2}, C_{2}>C_{1}$. H. Both forms apostatic. As the value of $C_{2}$ changes, the equilibrium point shifts $(x \rightarrow y)$ and is lost $(y \rightarrow z)$. I. Form 1 aposematic, form 2 apostatic with sigmoid frequency-fitness function, leading to multiple equilibria. See text for further discussion.

conclusion by Barrett (1976) flows from a subtly different definition of selection. Note that shifts in frequency-independent fitness components cause shifts in the equilibrium frequency, and frequency-independent fitness differences of sufficient magnitude preclude the existence of an equilibrium (fig. $2 \mathrm{~h}$ ).

\section{ConCURRENT APOStatic AND APOSEMAtic SElection}

In the model developed above each colour form is subject to a single form of frequency-dependent selection. Now suppose that each phenotype 
is subject to concurrent frequency-dependent selection from two sources, leading to the following fitnesses:

$\begin{array}{ccc}\text { Genotype } & \text { Frequency } & \text { Fitness } \\ A_{1}- & 1-q^{2} & C_{1}-s_{11}\left(1-q^{2}\right)-s_{12}\left(1-q^{2}\right) \\ A_{2} A_{2} & q^{2} & C_{2}-s_{21} q^{2}-s_{22} q^{2}\end{array}$

where $s_{11}, s_{12}, s_{21}$ and $s_{22}$ represent coefficients of frequency-dependent selection from two distinct sources on each colour form. Rearranged, the fitnesses take the form $C_{1}-\left(s_{11}+s_{12}\right)\left(1-q^{2}\right)$ and $C_{2}-\left(s_{21}+s_{22}\right) q^{2}$. Therefore $s_{11}+s_{12}$ and $s_{21}+s_{22}$ can be substituted for $s_{1}$ and $s_{2}$ in expressions (2-5) and the results derived above for case (1) can be applied, based on net $s$ values.

Provided that the magnitudes of the net $s$ values and the $C$ values are appropriately balanced, stable polymorphism may result from:

(a) net apostatic selection on both forms;

(b) net apostatic selection on one form combined with net aposematic selection on the other form;

(c) net apostatic selection on one form in the absence of frequency-dependent selection on the other form.

\section{FREQUENCY-DEPENDENT SELECTION WITH DISTINCT HETEROZYGOTES}

Many natural single-locus two-allele colour polymorphisms exhibit simple dominance, but some do not. When heterozygotes are distinct analysis becomes more complicated but work by Cockerham et al., (1972) permits ready solution of one fairly general case. Below is a slightly modified version of their "general dominance" model of frequency-dependent selection:

Genotype

Fitness in competition with

$$
\begin{array}{cccc} 
& A_{1} A_{1} & A_{1} A_{2} & A_{2} A_{2} \\
A_{1} A_{1} & C-s_{1} & C-(1-h) s_{1} & C \\
A_{1} A_{2} & C-(1-h) s_{1} & C-(1-h)^{2} s_{1}-h^{2} s_{2} & C-h s_{2} \\
A_{2} A_{2} & C & C-h s_{2} & C-s_{2}
\end{array}
$$

where $h$ is a measure of dominance between the frequency-dependent selection coefficients $s_{1}$ and $s_{2}$ and the other symbols are as in case (1) above (with $C_{1}=C_{2}=C$ ). Because the net fitness of each genotype depends on the frequencies of all three genotypes, selection is clearly frequencydependent. Not so obviously, this model leads directly to a generalised mathematical version of case (1) with $C_{1}=C_{2}$ because, assuming random mating and random competition among individuals with respect to genotype, the fitnesses of the genotypes are:

$\begin{array}{ccc}\text { Genotype } & \text { Frequency } & \text { Fitness } \\ A_{1} A_{1} & p^{2} & C-s_{1}\left[p^{2}+(1-h) 2 p q\right] \\ A_{1} A_{2} & 2 p q & C-(1-h) s_{1}\left[p^{2}+(1-h) 2 p q\right]-h s_{2}\left[q^{2}+h 2 p q\right] \\ A_{2} A_{2} & q^{2} & C-s_{2}\left[q^{2}+h 2 p q\right]\end{array}$


When $h=0$, allele $A_{1}$ is dominant and the fitnesses reduce to those of case (1). When $h=1$, allele $A_{1}$ is recessive. When $0<h<1$ (partial dominance), heterozygote fitness is a function of the frequency of all three phenotypes, and the fitness of each homozygote is a function of its own frequency and heterozygote frequency. This is an intuitively reasonable model for colour polymorphisms with heterozygotes of intermediate appearance that might be mistaken for either homozygote.

Cockerham et al., show that for $0<h<1$ a stable polymorphic equilibrium can occur only if both $s_{1}$ and $s_{2}$ are positive, a condition identical to that for the case of simple dominance. As above (6), net coefficients of frequency-dependence can be substituted for $s_{1}$ and $s_{2}$ so that $C-s_{1}$ becomes $C-\left(s_{11}+s_{12}\right)$, etc., and polymorphism for three aposematic forms is possible. When $h<0$ (underdominance) or $h>1$ (overdominance), two or three equilibria may occur, some stable and some not. Furthermore, the conditions for stability become much more complex and polymorphic equilibrium may be attained with $s_{1}$ and $s_{2}$ of opposite sign, an outcome that requires frequency-independent fitness differences in the case of simple dominance.

\section{LiMitATIONS OF THE MODELS}

For simplicity, the models assume a linear relationship between fitness and phenotype frequency. Natural apostatic and aposematic fitnessfrequency functions are unlikely to take such simple form (see Cook, 1965, and Fullick and Greenwood, 1979, on the probable sigmoid shape of apostatic functions). Small departures from linearity do not alter the conclusions reached above and even major departures may have little effect on conditions for equilibrium, provided that fitnesses change monotonically with frequency (Turner, 1980). However, if the frequency-independent fitness components of the phenotypes differ and the fitness of one phenotype declines monotonically with frequency while the fitness of the other rises, more than one equilibrium may occur (see Fig. $2 \mathrm{i}$ for an example) and the outcome of selection may depend on initial gene frequencies. This does not seriously compromise the conclusions, but it does underline the heuristic nature of the models.

Although the models are robust to departures from linearity, they apply only to cases in which all phenotypes occur in numbers high enough to educate predators through repeated encounters. Therefore they apply neither to very small populations nor to cases in which one phenotype occurs at very low frequency. Consequently, they do not deal with the fate of newly arising colour forms in existing monomorphic populations and they are not sufficient to predict the long term evolution of colour patterns under mixed apostatic and aposematic selection.

Two other limitations deserve brief mention. First, the models implicitly assume that all components of selection operate at the same stage of the life cycle. If the frequency-independent components act first, the results do not hold (Clarke and O'Donald, 1964; Turner, 1978). Second, though many natural colour polymorphisms involve three or more alleles or more than a single locus, the results are limited to single-locus two-allele cases (see Ginzburg, 1983, for a multi-allelic model). 


\section{Discussion}

Selection for warning colouration cannot by itself maintain stable colour polymorphism. This holds true whether or not there are also frequencyindependent selective differences between colour forms. Therefore, barring continued migration from an outside source, the stable presence of aposematic colour forms in a polymorphic population implies the existence of compensating frequency-dependent selection. As shown above, concurrent apostatic selection can stabilise colour polymorphisms for one, two or three aposematic forms determined by two alleles at a single locus.

What, then, are the circumstances in which polymorphism for warning colouration might occur? First, polymorphism for well-defended aposematic forms and ill-defended cryptic forms is possible. No cases of precisely this sort have been documented in nature (Pasteels and Grégoire, 1983) but there are at least two instances in which aposematic colour forms are better defended than alternative colour forms of the same species. Marsh and Rothschild (1974) report that the aberrant variety lutea of the moth Callimorpha dominula is considerably less toxic than the aposematic typical form, and Hensel and Brodie (1976) report that red-marked aposematic forms of the salamander Plethodon jordani are unpalatable whereas an all-black (allopatric) form is not.

It is more likely that aposematic polymorphism will occur when some predators find a prey species desirable and select apostatically, while other predators find it undesirable and select for warning colouration. Predator heterogeneity, which would be most pronounced for moderately welldefended prey, could take the form of interspecific (Edmunds, 1969) or intraspecific (Greenwood et al., 1981) variation in prey preference. Based on this criterion, a number of terrestrial insects exposed to avian predation are likely candidates for aposematic polymorphism. For example, many ladybird beetles are polymorphic for colour forms that appear to be aposematic. Although these beetles contain repellent alkaloids, birds do attack and eat many species (Muggleton, 1978) and at least one highly polymorphic species (Adalia bipunctata) seems to elicit very different predatory responses from different individual quail (Pasteels et al., 1973). This suggests that a combination of modest chemical defences plus apostatic selection may be the basis for some ladybird polymorphisms.

Similarly, a number of otherwise ill-defended jumping insects seem to be polymorphic for warning colouration. Though jumping is a relatively mild form of anti-predator defence, it can lead to aposematic selection (Gibson, 1980) and may provide a basis for aposematic polymorphism. Polymorphic jumping species with apparently aposematic forms include the spittlebug Philaenus spumarius (Thompson, 1973), the planthopper Phylloscelis atra (Ball, 1930), grouse locusts of the genera Apotettix and Paratettix (Nabours, 1929), and a number of flea beetles (Horn, 1889). Although the flea beetles (Alticinae) belong to a family (Chrysomelidae) characterised by repellent secretions, their chemical defences seem to be reduced or absent (Deroe and Pasteels, 1982).

In other cases polymorphism for warning colouration occurs in species that appear to be better defended (though still subject to predation). This category includes the unpalatable butterfly Danaus chrysippus (Edmunds, 1969; Owen, 1970; Greenwood et al., 1981) and the bumblebees Bombus 
rufocinctus and B. melanopygus (Plowright and Owen, 1980). It may also include some populations of the extraordinarily polymorphic poison-dart frog Dendrobates pumilis (Daly and Myers, 1967; Myers and Daly, 1983). Both $D$. chrysippus and $D$. pumilis seem to exhibit substantial variation in toxicity and male bumblebees cannot sting, factors that could contribute to heterogeneity in predator response to these species.

I have cited cases in which the models developed above might apply. Workers familiar with some of the species in question have offered alternative explanations (e.g., Owen, 1970; Plowright and Owen, 1980) but direct evidence for or against the hypothesis that a given aposematic polymorphism is stabilized by apostatic selection will be difficult to gather. This is because under balanced apostatic and aposematic selection fitness differences may diminish to zero at equilibrium and very low levels of predation may maintain polymorphism, levels so low as to be unmeasurable in practice. Nevertheless, I hope these models will stimulate closer field and laboratory study of many striking and perplexing colour polymorphisms that have so far eluded convincing explanation.

Acknowledgements. I am indebted to Lisa Brooks for the stability analysis in Appendix I. I also thank her and an anonymous reviewer for helpful comments.

\section{APPENDIX I. LOCAL STABILITY OF EQUILIBRIUM}

At equilibrium $\Delta q=0$. A polymorphic equilibrium $\hat{q}$ is locally stable if an arbitrarily small positve or negative displacement of $q$ from $\hat{q}$ is followed by a return to $\hat{q}$, either directly or through damped oscillations. Following Lewontin (1958) and Turner (1980), an equilibrium is stable if:

$$
-2<\left.\frac{d \Delta q}{d q}\right|_{\hat{q}}<0
$$

If the derivative is greater than 0 , perturbation of $q$ leads to monotonic divergence from $\hat{q}$ and the equilibrium is unstable. If the derivative is less than $-2, q$ will oscillate about $\hat{q}$ with increasing amplitude until one allele is lost. If it lies between -2 and $-1, q$ will converge on $\hat{q}$ through damped oscillations, and if it lies between -1 and $0, q$ will simply return to $\hat{q}$.

From (3):

$$
\frac{d \Delta q}{d q}=\frac{d\left[p q^{2}\{\} / \bar{W}\right]}{d q}
$$

where \{\}$=\left[C_{2}-s_{2} q^{2}\right]-\left[C_{1}-s_{1}\left(1-q^{2}\right)\right]$, the term in curly brackets in the numerator of (3), and $\bar{W}$ is the denominator of (3). Taking the derivative, this becomes:

$$
\frac{d \Delta q}{d q}=\frac{p q^{2}}{\bar{W}} \frac{d\{\}}{d q}+\frac{\{\} d\left[p q^{2} / \bar{W}\right]}{d q} .
$$

At $\hat{q}$ the fitnesses of the phenotypes are equal so that \{\}$=0$, the term on the right drops out, and:

$$
\left.\frac{d \Delta q}{d q}\right|_{\hat{q}}=\left.\frac{p q^{2}}{\bar{W}} \frac{d\left\{\left[C_{2}-s_{2} q^{2}\right]-\left[C_{1}-s_{1}\left(1-q^{2}\right)\right]\right\}}{d q}\right|_{\hat{q}}
$$


which reduces to:

$$
\left.\frac{d \Delta q}{d q}\right|_{\hat{q}}=-2 \hat{p} \hat{q}^{3}\left(s_{1}+s_{2}\right) / \hat{W} .
$$

Therefore, the equilibrium is stable if:

$$
-2<-2 \hat{p} \hat{q}^{3}\left(s_{1}+s_{2}\right) / \hat{W}<0 .
$$

Since $\hat{p}, \hat{q}$ and $\hat{W}$ are positive, this holds true so long as:

$$
0<s_{1}+s_{2}<\hat{W} /\left[\hat{p} \hat{q}^{3}\right] \text {. }
$$

The right hand condition is deceptively simple, since $\hat{p}, \hat{q}$ and $\hat{W}$ are functions of $s_{1}$ and $s_{2}$.

Note that when the fitness of each form is expressed as a function of $1-q^{2}$ (figs. 1 and 2), the sum $s_{1}+s_{2}$ is equal to the slope of the fitnessfrequency function of form 2 minus the slope of the fitness-frequency function of form 1. For a stable equilibrium, this difference must be positive but not too large. Each allele must be at advantage when rare, but the advantage cannot be too great.

\section{REFERENCES}

AyAla, F. J. 1978. The mechanisms of evolution. Sci. Amer., 239, 3, 56-69.

AYAla, F. AND CAMPBEll, C. A. 1974. Frequency-dependent selection. Ann. Rev. Ecol. Syst., $5,115-138$.

BALL, E. D. 1930. The toadhoppers of the genus Phylloscelis Germ. (Rhynchota-Fulgoridae). Can. Entomol., 62, 193-195.

BARRETT, J. A. 1976. The maintenance of non-mimetic forms in a dimorphic Batesian mimic species. Evolution, 30, 82-85.

CLARKE, B. 1979. The evolution of genetic diversity. Proc. Roy. Soc., Lond. (Biol.), 205, 453-474.

CLARKE, B. AND O'DONALD, P. 1964. Frequency-dependent selection. Heredity, 19, 201-206.

COCKERHAM, C. C., BURROWS, P. M., YOUNG, S. S. AND PROUT, T. 1972. Frequency-dependent selection in randomly mating populations. Amer. Natur., 106, 493-515.

COOK, L. M. 1965. A note on apostasy. Heredity, 20, 631-636.

DALY, J. W. AND MYERS, C. W. 1967. Toxicity of Panamanian poison frogs (Dendrobates): some biological and chemical aspects. Science, 156, 970-973.

DEROE, C. AND PASTEELS J. M. 1982. Distribution of adult defence glands in chrysomelids (Coleoptera: Chrysomelidae) and its significance in the evolution of defense mechanisms within the family. J. Chem. Ecol., 8, 67-82.

EDMUNDS, M. 1969. Polymorphism in the mimetic butterfly Hypolimnas misippus L. in Ghana. Heredity, 24, $281-302$.

FISHER, R. A. 1958. The Genetical Theory of Natural Selection. 2nd Ed. Dover, N.Y.

FULLICK, T. G. AND GREENWOOD, J. J. D. 1979. Frequency-dependent food selection in relation to two models. Amer. Natur., 113, 762-765.

GIBSON, D. O. 1980. The role of escape in mimicry and polymorphism: I. The response of captive birds to artificial prey. Biol. J. Linn. Soc., Lond. 14, 201-214.

GINZBURG, L. R. 1983. Theory of Natural Selection and Population Growth. Benjamin/Cummings, Menlo Park.

GREENWOOD, J. J. D., WOOD, E. M. AND BATCHELOR, S. 1981. Apostatic selection of distasteful prey. Heredity, 47, 27-34.

HENSEL, J. L. AND BRODIE, E. D. 1976. An experimental study of aposematic coloration in the salamander Plethodon jordani. Copeia, 1976, 59-65.

HODEK, I. 1973. Biology of the Coccinellidae. Academia, Prague.

HORN, G. H. 1889. A synopsis of the Halticini of boreal America. Trans. Entomol. Soc. Amer., $16,163-320$.

LEWONTIN, R. C. 1958. A general method for investigating the equilibrium of gene frequency in a population. Genetics, $43,419-434$. 
MARSH, N. AND ROTHSCHILD, M. 1974. Aposematic and cryptic Lepidoptera tested on the mouse. J. Zool., Lond., 174, 89-112.

MUGGleton, J. 1978. Selection against the melanic morphs of Adalia bipunctata (two-spot ladybird): a review and some new data. Heredity, 40, 269-280.

MYERS, C. W AND DALY, J. w. 1983. Dart-poison frogs. Sci. Amer., 248, 2, 120-133.

NABOURS, R. K. 1929. The genetics of the Tettigidae (Tetriginae). Bibliogr. Genet., 5, 27-104.

O'DONALD, P. 1980. A general analysis of genetic models with frequency-dependent mating. Heredity, 44, 309-320.

OWEN, D. F. 1970. Mimetic polymorphism and the palatability spectrum. Oikos, 21, 333-336.

PASTEELS, J, M., DEROE, C., TIRSCH, B., BRAEKMAN, J. C., DALOZE, J. C. AND hOOTELE, D. 1973. Distribution et activités des alcaloïdes défensifs des Coccinellidae. J. Insect Physiol., 19, $1771-1784$.

PASTEELS, J. M. AND GREgoIRE, J.-C. 1983. The chemical ecology of defence in arthropods. Ann. Rev. Entomol., 28, 263-289.

PLOWRIGHT, R. C. AND OWEN, R. E. 1980. The evolutionary significance of bumblebee color patterns: a mimetic interpretation. Evolution, 34, 622-637.

THOMPSON, v. 1973. Spittlebug polymorphic for warning coloration. Nature, 242, 126-128.

THOMPSON, v. 1974. Reply to: Spittlebug morph mimics avian excrement. Nature, 250, 352-353.

TURNER, J. R. G. 1978. Why male butterflies are non-mimetic: natural selection, sexual selection, group selection, modification and sieving. Biol. J. Linn. Soc., Lond., 10, 385-432.

TURNER, J. R. G. 1980. Oscillations of frequency in Batesian mimics, hawks and doves, and other simple frequency dependent polymorphisms. Heredity, 45, 113-126.

WRIGHT, S. 1955. Classification of some factors of evolution. Cold Spring Harbor Symp. Quant. Biol, 20, 16-24. 